\title{
Extracting Meaningful Slopes from Terrain Contours
}

\author{
Maciej Dakowicz and Christopher Gold \\ Department of Land Surveying and Geo-Informatics \\ Hong Kong Polytechnic University, Hung Hom, Kowloon, Hong Kong \\ Tel: (852) 2766-5955; Fax: (852) 2330-2994 \\ maciej.dakowicz@dtm.prv.pl, christophergold@voronoi.com
}

\begin{abstract}
Good quality terrain models are becoming more and more important, as applications such as runoff modelling are being developed that demand better surface orientation information than is available from traditional interpolation techniques. A consequence is that poor-quality elevation grids must be massaged before they provide useable runoff models. Rather than using direct data acquisition, this project concentrated on using available contour data because, despite modern techniques, contour maps are still the most available form of elevation information. Recent work on the automatic reconstruction of curves from point samples, and the generation of medial axis transforms (skeletons) has greatly helped in expressing the spatial relationships between topographic sets of contours. With these techniques the insertion of skeleton points into a TIN model guarantees the elimination of all "flat triangles" where all three vertices have the same elevation. Additional assumptions about the local uniformity of slopes give us enough information to assign elevation values to these skeleton points. In addition, various interpolation techniques were compared using the enriched contour data. Examination of the quality and consistency of the resulting maps indicates the required properties of the interpolation method in order to produce terrain models with valid slopes. The result provides us with a surprisingly realistic model of the surface - that is, one that conforms well to our subjective interpretation of what a real landscape should look like.
\end{abstract}

\section{Introduction}

This paper concerns the generation of interpolated surfaces from contours. While this topic has been studied for many years [5], [6], [7], [11], [13], [15], the current project is interesting for a variety of reasons. Firstly, contour data remains the most readily available data source. Secondly, valid theorems for the sampling density along the contour lines have only recently been discovered [1]. Thirdly, the same publications provide simple methods for generating the medial axis transform, or skeleton, which definitively solves the "flat triangle" problem (which often occurs when triangulating contour data) by inserting additional points from this skeleton. Fourthly, the problem of assigning elevation values to these additional ridge or valley points can be resolved, using the geometric properties of this skeleton, in ways that may be associated with the geomorphological form of the landscape. In addition, comparisons of the methods used in a variety of weighted-average techniques throw light on the 
key components of a good weighted-average interpolation method, using threedimensional visualization tools to identify what should be "good" results - with particular emphasis being placed on reasonable slope values, and slope continuity. This last is often of more importance than the elevation itself, as many issues of runoff, slope stability and vegetation are dependent on slope and aspect - but unfortunately most interpolation methods can not claim satisfactory results for these properties. The techniques developed here are based on the simple point Voronoi diagram and the dual Delaunay triangulation.

\section{Generation of Ridge and Valley Lines}

Amenta et al. [1] examined the case where a set of points sampled from a curve, or polygon boundary, were triangulated, and then attempted to reconstruct the curve. They showed that this "crust" was formed from the triangle edges that did not cross the skeleton, and that if the sampling of the curve was less than 0.25 of the distance to the skeleton the crust was guaranteed to be correct. Gold [9] and Gold and Snoeyink [10] simplified their algorithm for extraction of the crust, showing that, in every Delaunay/Voronoi edge pair, either the Delaunay edge could be assigned to the crust or else the dual Voronoi edge could be assigned to the skeleton. The Delaunay edge belongs to crust when there exists a circle through its two vertices that does not contain either of its associated Voronoi vertices; if not then the corresponding Voronoi edge belongs to the skeleton. Skeleton points may be inserted into the original diagram, or not, as needed.

In our particular case the data is in the form of contour lines that we assume are sufficiently well sampled - perhaps derived from scanned maps. Despite modern satellite imaging, much of the world's data is still in this form. An additional property is not sufficiently appreciated - they are subjective, the result of human judgement at the time they were drawn. Thus they are clearly intended to convey information about the perceived form of the surface at a particular scale - and it would be desirable to preserve this, as derived ridges and valleys.

Fig. 1a shows our raw data set (which is completely imaginary), and Fig. 1b shows the resulting crust, which reconstructs the contour lines and the skeleton. Fig. 1c shows the crust and only those skeleton points that provide unique information - ridge and valley lines that separate points on the same contour, rather than merely those points that separate adjacent contours. Aumann et al. [3] produced somewhat similar results by raster processing.

Fig. 2a shows a close-up of the test data set, illustrating a key point of Amenta et a.l's work: if crust edges (forming the contour boundary) may not cross the skeleton, then inserting the skeleton points will break up non-crust triangle edges. In particular, if the skeletons between different contours are ignored, then insertion of the remaining branch skeleton points will eliminate all "flat triangles" formed from points of the same elevation. Thus ridge and valley lines are readily generated automatically. The same is true in the case of closed summits (Fig. 2b). The challenge is to assign meaningful elevation values to skeleton points. 

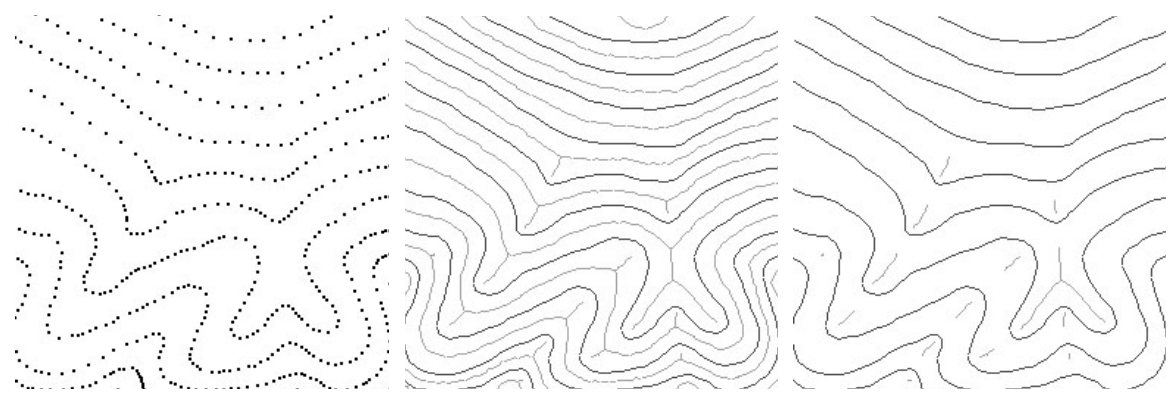

Fig. 1. Contours - a) data points; b) crust and skeleton; c) crust and skeleton branches
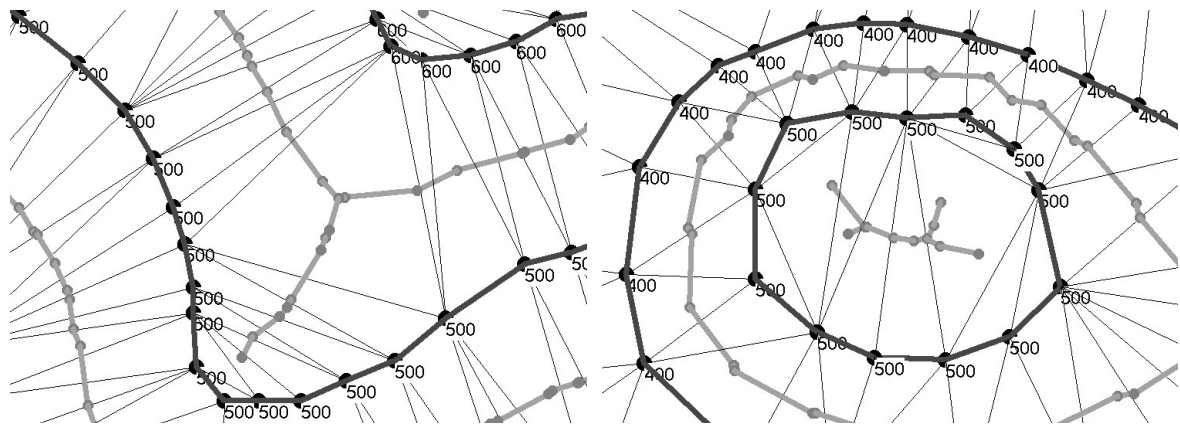

Fig. 2. Skeleton and "flat triangles" - a) ridge; b) summit

Two techniques have been developed for this, each with its own physical interpretation. The first, following Thibault and Gold [14], uses Blum's [4] concept of height as a function of distance from the curve or polygon boundary, with the highest elevations forming the crest at the skeleton line. This is illustrated in Figs. 3a and 3b, where points on a simple closed curve are used to generate the crust and skeleton.
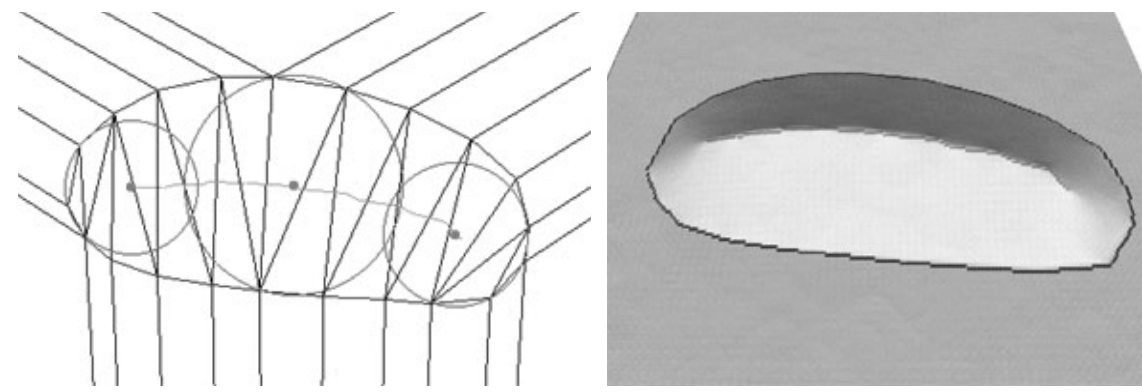

Fig. 3. Triangulation of a summit - a) skeleton and circumcentres; b) elevation model after adding skeleton vertices with assigned height values

In Fig. 3a, the circumcentres of the skeleton points are given a height above the previous contour level equal to the circumradius. The resulting interpolated model is shown in Fig. 3b. This model is based on the idea that all slopes are identical, and 
thus the radius is proportional to the height of the skeleton point. Of course, in the case of a real summit as in Fig. 2b, the slope would initially be unknown, and would be estimated using circumradii from the next contour level down - see [14].

In the case of a ridge or valley, the circumradius may also be used, as in Fig. 4a, to estimate skeleton heights based on the hypothesis of equal slopes. The larger circle, at the junction of the skeleton branches, has a known elevation - half way between the contours - and may be used to estimate the local slope. The elevation of the centre of the smaller circle is thus based on the ratio of the two radii. For more details see [14].

While this method is always available, it is not always the preferred solution where constant slope down the drainage valley, rather than constant valley-side slope, is more appropriate. In a second approach, illustrated in Fig. 4b, the line of the valley is determined by searching along the skeleton, and heights are assigned based on their relative distance along this line. This may be complicated where there are several valley branches - in which case the longest branch is used as the reference line. This involves careful programming of the search routines, although the concept is simple. In practice, an automated procedure has been developed, which uses the valley length approach where possible, and the side-slope method when no valley head can be detected, such as at summits and passes.

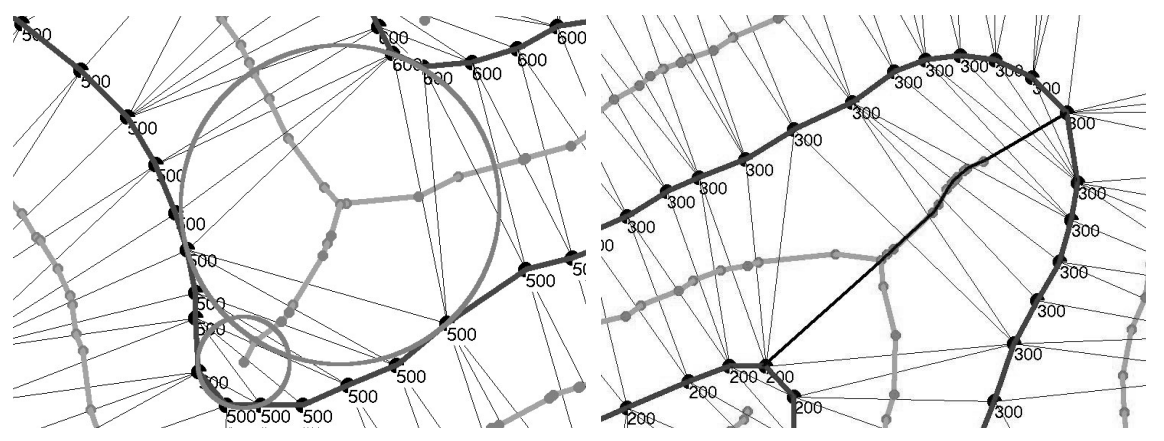

Fig. 4. Estimating skeleton heights - a) from circumradii; b) from valley length

\section{Components of an Interpolation Model}

On the basis of a sufficient set of data points, we now wanted to generate a terrain model with satisfactory elevations and slopes, as the basis of a valid rainfall runoff model. Our approach was to interpolate a height grid over the test area, and to view this with an appropriate terrain visualization tool. To obtain perspective views we used Genesis II, available from www.geomantics.com. Vertical views were generated using version 5 of the Manifold GIS, available from www.Manifold.net. We feel that $3 \mathrm{D}$ visualization has been under-utilized as a tool for testing terrain modelling algorithms, and the results are often more useful than a purely mathematical or statistical approach. 
We have restricted ourselves to an evaluation of several weighted-average methods, as there are a variety of techniques in common that can be compared. All of the methods were programmed by ourselves - which left out the very popular Kriging approach, as too complicated, and not necessarily better. Nevertheless, many aspects of this study apply to this method as well, since it is a weighted-average method with the same problems of neighbour selection, etc., as the methods we attempted

In general, we may ask about three components of a weighted-average interpolation method. Firstly: what is the weighting process used? Secondly: what is the set of neighbours used to obtain the average? Thirdly: what is the elevation function being averaged? (Often it is the data point elevation alone, but sometimes it is a plane through the data point incorporating slope information as well.)

One simple weighted-average model is the triangulation, using the Delaunay triangulation. Fig. 5 shows the result, including the crust and skeleton draped over the terrain. The flat triangles are readily seen.. Fig. 6 shows the improved model when estimated skeleton points are added, and all flat triangles are removed.
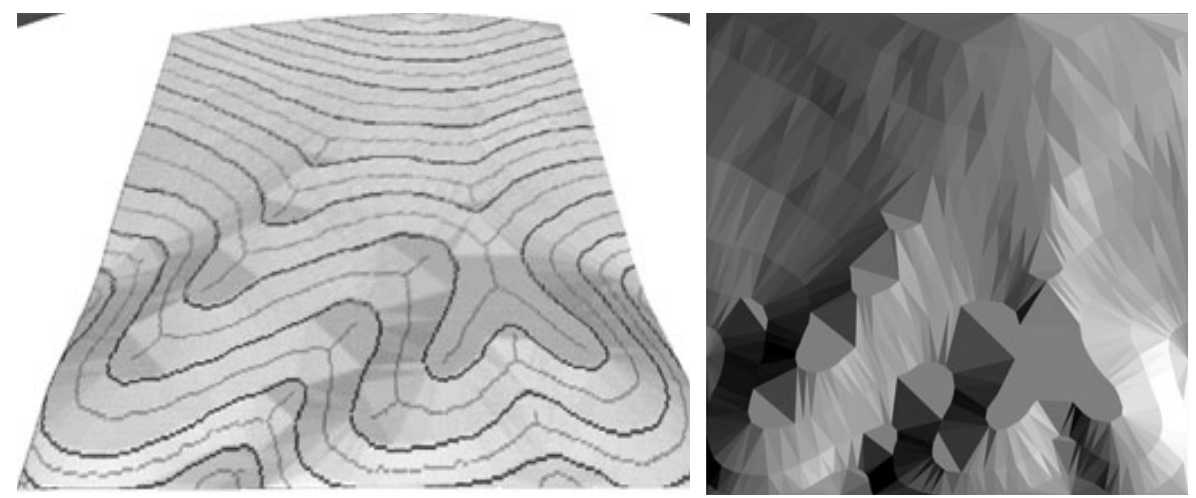

Fig. 5. Interpolation from Delaunay triangulation - a) perspective view; b) vertical view

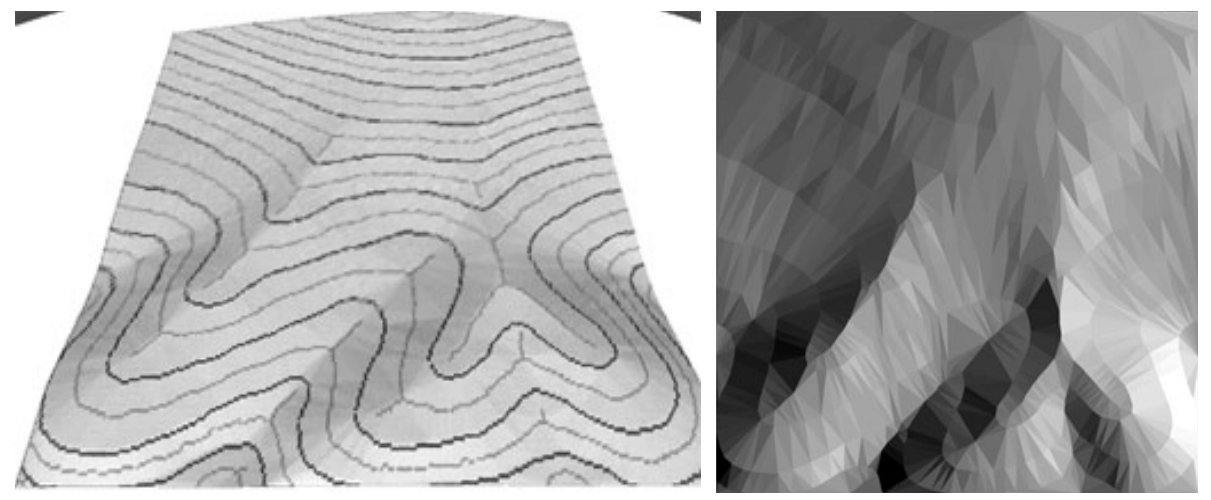

Fig. 6. Adding skeleton points to Fig. 5 - a) perspective view; b) vertical view 
The other weighted average models that were tested were the traditional gravity model, and the more recent "area-stealing" or "natural neighbour" or perhaps more properly "Sibson" interpolation methods ([8], [12], [16]).

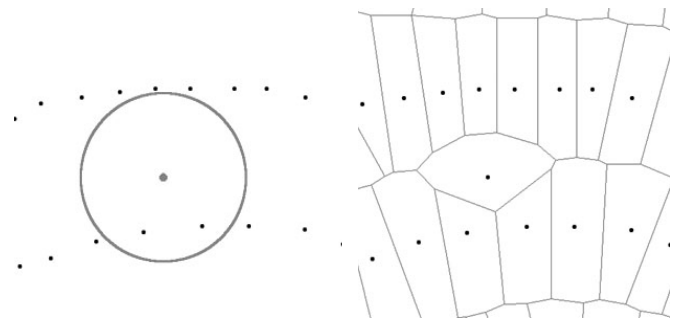

Fig. 7. Neighbour selection a) using a counting circle; b) using Voronoi neighbours

In the case of the gravity model the weighting of each data point used is inversely proportional to the square of the distance from the data point to the grid node being estimated, although other exponents have been used. There is no obvious set of data points to use, so one of a variety of forms of "counting circle" is used, as in Fig. 7a. When the data distribution is highly anisotropic there is considerable difficulty in finding a valid counting circle radius. Fig. 8 shows the resulting surface for a radius of about a quarter of the map. Data points form bumps or hollows. If the radius is reduced there may be holes in the surface where no data is found within the circle. If the radius is increased the surface becomes somewhat flattened, but the bumps remain. The result depends on the radius, and other selection properties, being used. Clearly, in addition, estimates of slope would be very poor, and very variable.

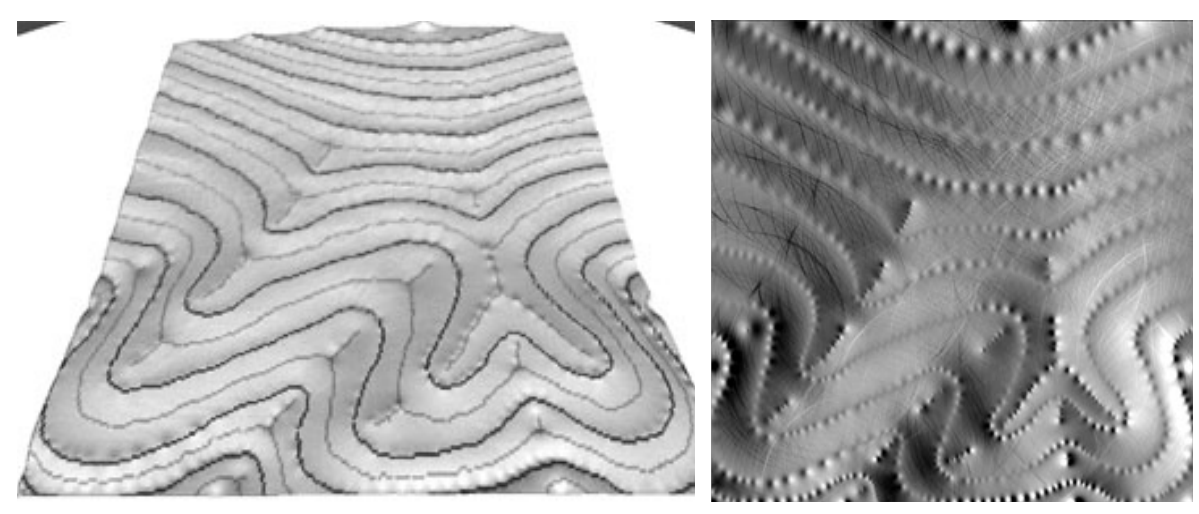

Fig. 8. Interpolation using the gravity model - a) perspective view; b) vertical view

The Sibson method is based on the idea of inserting each grid point temporarily into the Voronoi diagram of the data points, and measuring the area stolen from each of a well-defined set of neighbours. These stolen areas are the weights used for the weighted-average. The method is particularly appropriate for poor data distributions, as illustrated in Fig. $7 b$, as the number of neighbours used is well defined, but dependent on the data distribution. Fig. 9 shows the results of using Sibson 
interpolation. The surface behaves well, but is angular at ridges and valleys. Indeed, slopes are discontinuous at all data points (Sibson [12]). One solution is to re-weight the weights, so that the contribution of any one data point not only becomes zero as the grid point approaches it, but the slope of the weighting function approaches zero also (Gold [8]). Fig. 10 shows the effect of adding this smoothing function. While the surface is smooth, the surface contains undesirable "waves" - indeed, applying this function gives a surface with zero slope at each data point.
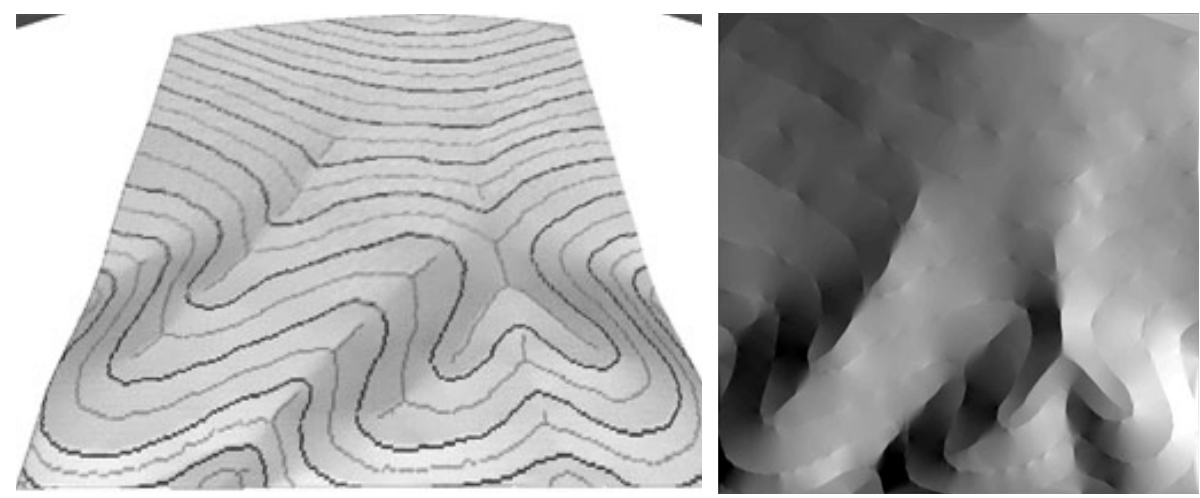

Fig. 9. Sibson interpolation - a) perspective view; b) vertical view
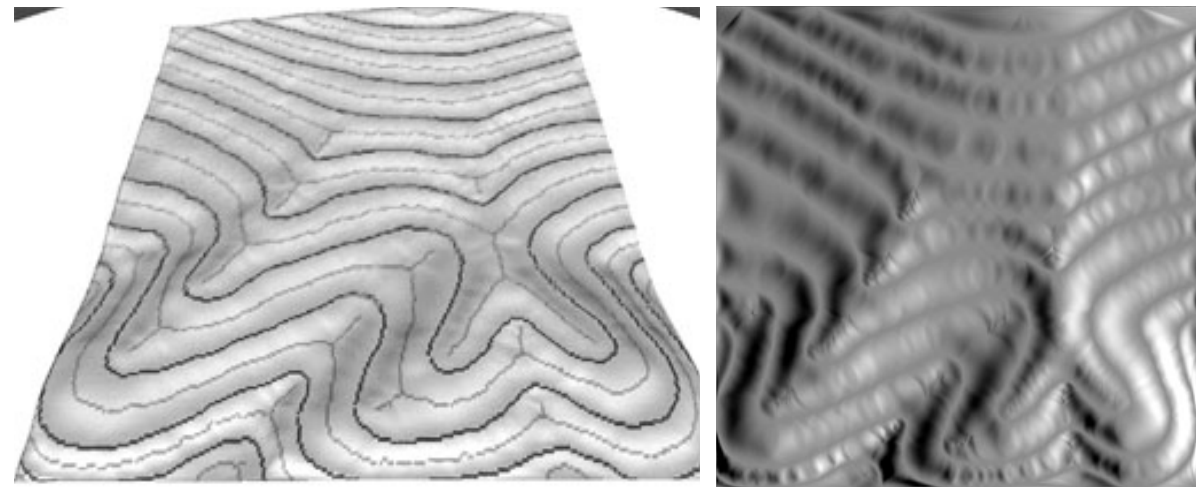

Fig. 10. Adding smoothing to Fig. 9 - a) perspective view; b) vertical view

\section{Slopes - The Ignored Factor}

This brings us to a subject often ignored in selecting a method for terrain modelling the slope of the generated surface. In real applications, however, accuracy of slope is often more important than accuracy of elevation - for example in runoff modelling, erosion. Clearly an assumption of zero slope, as above, is inappropriate. However, in our weighted-average operation we can replace the height of a neighbouring data 
point by the value of a function defined at that data point - probably a planar function involving the data point height and local slopes. Thus at any grid node location we find the neighbouring points and evaluate their planar functions for the $(x, y)$ of the grid node. These $\mathrm{z}$ estimates are then weighted and averaged as before.

Fig. 11 shows the result of using Sibson interpolation with data point slopes. The form is good, but slight breaks in slope can be seen at contour lines. When using smoothing and slope information together, the surface is smooth, but has unwanted oscillations, see Fig. 12. Clearly an improved smoothing function is desirable to eliminate these side-effects.

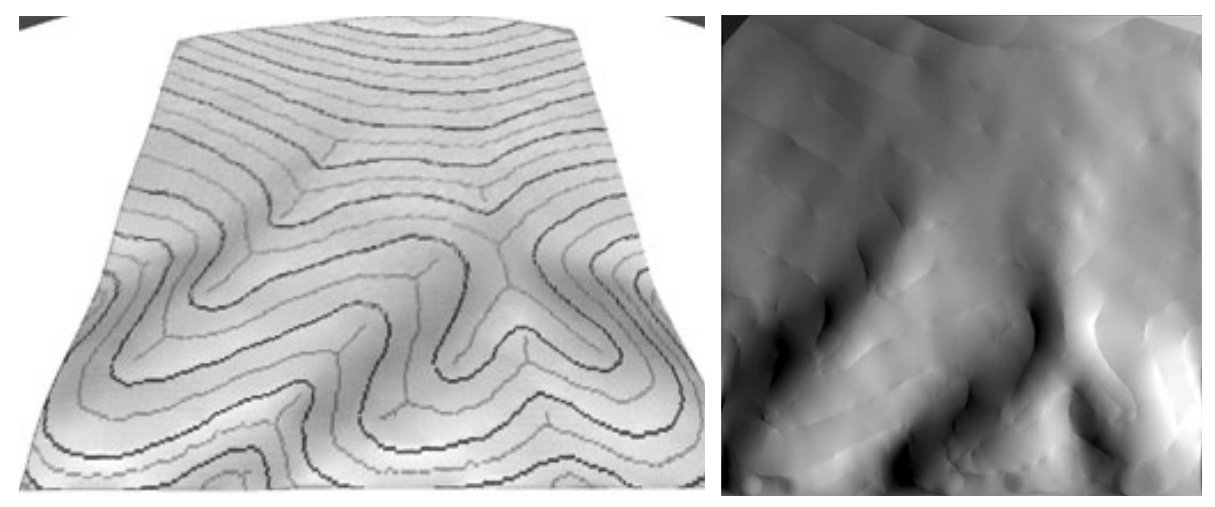

Fig. 11. Sibson interpolation using slopes at data points - a) perspective; b) vertical view
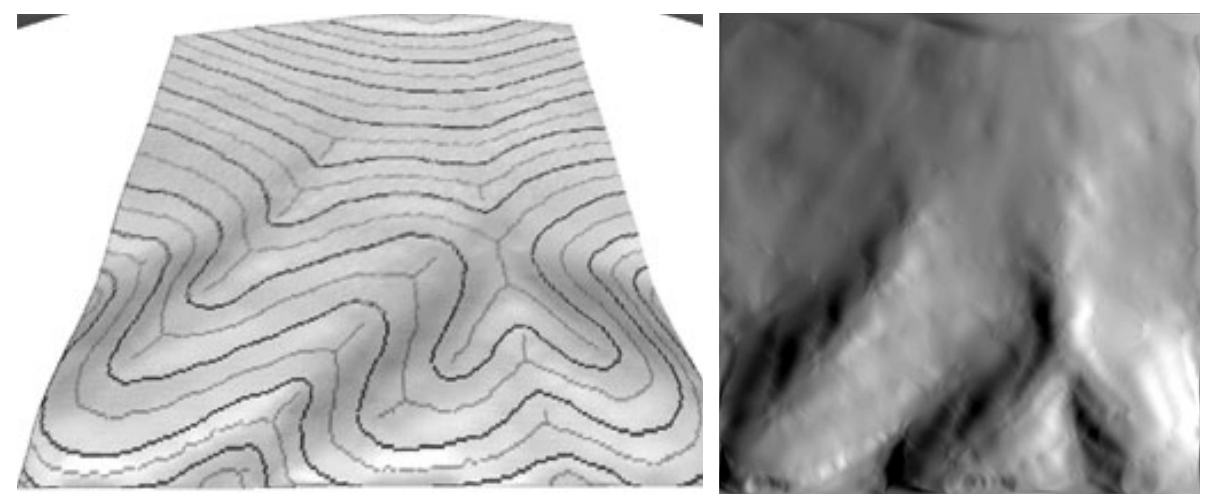

Fig. 12. Sibson interpolation using slopes and smoothing function at data points a) perspective view; b) vertical view

Adding slopes to the simple TIN model (i.e. using the position in the triangle to provide the weights) produced results that were almost as good as the Sibson method when the sample points were closely spaced along the contours. However, the Sibson method is much superior for sparser data, or where the points do not form contour lines. The gravity model does not provide particularly good slope estimates, but even here including the data point slope function produces a significant improvement. 


\section{Proposed methodology and conclusions}

For the common problem of deriving surfaces from contours, we propose a general approach:

1. Generate skeleton points along the ridges, valleys, pits, summits and passes by the method of Aumann et al. [3] or of Thibault and Gold [14].

2. Assign elevations to these skeleton points by the methods described here, or other suitable techniques.

3. Eliminate flat triangles by the insertion of these skeleton points into the original TIN.

4. Estimate slope information at each data point by any appropriate technique.

5. Perform weighted-average interpolation using the previously estimated slope information. Avoid methods, such as the gravity model, with exponentially large close-range weightings, and avoid neighbour selection techniques which require user-specified parameters, such as counting-circle radius.

Surprisingly, mathematically guaranteed slope continuity is not usually critical, although we are continuing to work on an improved smoothing function that guarantees both slope continuity and minimum curvature - probably based on the work of Anton et al. [2]. Nevertheless, the moral is clear: both for finding adjacent points and for skeleton extraction, a consistent definition of neighbourhood is essential for effective algorithm development.

We conclude with another imaginary example. Fig. 13a shows four small hills defined by their contours, modelled by a simple triangulation. Fig. 13b shows the result using Sibson interpolation, slopes and skeletons. Skeleton heights were obtained using circumcircle ratios, as no valley-heads were detected. While our evaluation was deliberately subjective, we consider that our results in this case, as with the previous imaginary landform, closely follow the perceptual model of the original interpretation. Thus, for the reconstruction of surfaces from contours, we believe that our methods are a significant improvement on previous work.
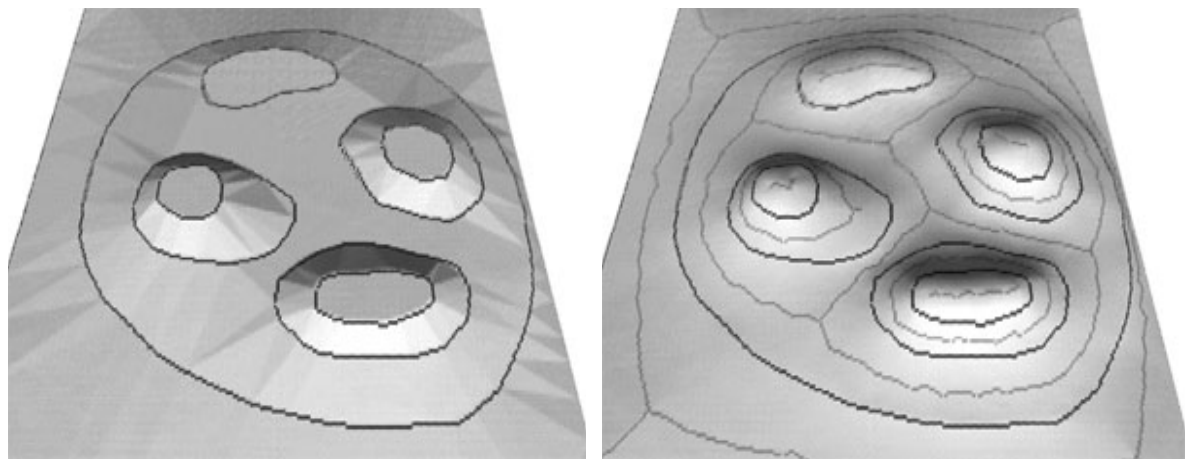

Fig. 13. Triangulation of several small hills - a) triangle based interpolation;

b) Sibson interpolation with slopes 


\section{Acknowledgments}

The work described in this paper was substantially supported by a grant from the Hong Kong Polytechnic University (Project No. PolyU A-PB79).

\section{References}

1. Amenta, N. Bern, M. and Eppstein, D. (1998) "The crust and the beta-skeleton: combinatorial curve reconstruction", Graphical Models and Image Processing, 60, 125-135.

2. Anton, F. Gold, C.M. and Mioc, D. (1998) "Local coordinates and interpolation in a Voronoi diagram for a set of points and line segments", Proceedings 2nd Voronoi Conference on Analytic Number Theory and Space Tillings, Kiev, Ukraine, 9-12.

3. Aumann, G. Ebner, H. and Tang, L. (1991) "Automatic derivation of skeleton lines from digitized contours", ISPRS Journal of Photogrammetry and Remote Sensing, 46, 259-268.

4. Blum, H. (1967) "A transformation for extracting new descriptors of shape", In: Whaten Dunn, W. (eds.), "Models for the Perception of Speech and Visual Form", 153-171, MIT Press.

5. Crain, I.K. (1970) "Computer interpolation and contouring of two-dimensional data: a review", Geoexploration, 8, 7x-86.

6. Davis, J. C. (1973) "Statistics and data analysis in geology", 313, New York, John Wiley and Sons.

7. Dayhoff, M.O. (1963) "A contour map program for X-ray crystallography", Communications of the Association for Computing Machinery, 6, 620-622.

8. Gold, C.M. (1989) "Chapter 3 - Surface interpolation, spatial adjacency and GIS", In: Raper, J. (eds.), "Three Dimensional Applications in Geographic Information Systems", 21-35, Taylor and Francis, Ltd., London.

9. Gold, C.M. (1999) "Crust and anti-crust: a one-step boundary and skeleton extraction algorithm", Proceedings of the ACM Conference on Computational Geometry, Miami, Florida, 189-196.

10. Gold, C. M. and Snoeyink, J. (2001) “A one-step crust and skeleton extraction algorithm”, Algorithmica, 30, 144-163.

11. Peucker, T.K. (1978) "The triangulated irregular network", Proceedings, Digital Terrain Model Symposium, American Society of Photogrammetry, St. Louis.

12. Sibson, R. (1980) "A Vector Identity for the Dirichlet Tessellation", Math. Proc. Cambridge Philos. Soc., 87, 151-155.

13. Sibson, R. (1982) "A brief description of natural neighbour interpolation”, In: Bamett, V. (eds), "Interpreting Multivariate Data", 21-36, John Wiley and Sons, London.

14. Thibault, D. and Gold, C.M. (2000) "Terrain Reconstruction from Contours by Skeleton Construction", GeoInformatica, 4, 349-373.

15. Walters, R.F. (1969) “Contouring by machine: a users' guide”, American Association of Petroleum Geologists, Bulletin, 53, 2324-2340.

16. Watson, D.F. and Philip, G.M. (1987) "Neighborhood-based interpolation", Geobyte, 2, 12-160. 